\title{
What can we learn from HF signal scattered from a discrete arc?
}

\author{
E. Séran ${ }^{1}$, M. Godefroy ${ }^{1}$, K. Kauristie ${ }^{2}$, J.-C. Cerisier ${ }^{1}$, J.-J. Berthelier ${ }^{1}$, M. Lester ${ }^{3}$, and L.-E. Sarri ${ }^{4}$ \\ ${ }^{1}$ LATMOS, 4 Avenue de Neptune, 94100 Saint-Maur, France \\ ${ }^{2}$ SRU/FMI, Finland \\ ${ }^{3} \mathrm{DPA} /$ Leicester University, UK \\ ${ }^{4}$ SSC/Esrange, Sweden
}

Received: 10 October 2008 - Revised: 25 February 2009 - Accepted: 6 April 2009 - Published: 4 May 2009

\begin{abstract}
We present observations of a discrete southward propagating arc which appeared in the mid-night sector at latitudes equatorward of main substorm activity. The arc observations were made simultaneously by the ALFA (Auroral Light Fine Analysis) optical camera, the SuperDARNCUTLASS HF radar and the Demeter satellite during a coordinated multi-instrumental campaign conducted at the KEOPS/ESRANGE site in December 2006. The SuperDARN HF signal which is often lost in the regions of strong electron precipitation yields in our case clear backscatter from an isolated arc of weak intensity. Consequently we are able to study arc dynamics, the formation of meso-scale irregularities of the electron density along the arc, compare the arc motion with the convection of surrounding plasma and discuss the contribution of ionospheric ions in the arc erosion and its propagation.
\end{abstract}

Keywords. Ionosphere (Auroral ionosphere; Electric fields and currents; Particle acceleration)

\section{Introduction}

The magnetospheric origin of individual auroral arcs has been discussed widely in the literature since the pioneering scenario originally suggested by Boström (1964). In this picture which has been later revised e.g. by Rothwell et al. (1991) an auroral arc is associated with a system of two current circuits comprised of field aligned currents (FACs) and magnetospheric and ionospheric currents (hereafter called M-I current circuits). In system II the magnetospheric (ionospheric) currents are radial (meridional) while in system I they are azimuthal. As auroral arcs are associated

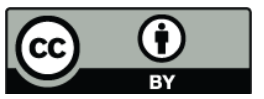

Correspondence to: E. Séran (elena.seran@latmos.ipsl.fr) with field aligned current sheets it is natural to assume a coupling between arc generator mechanisms and those processes at the magnetospheric equator which cause field aligned currents. Gradients in the plasma pressure, magnetic tension forces and shears in the convection flow are examples of important factors causing divergence of magnetospheric currents to FACs. The auroral arc at the ionospheric end and acceleration region processes are often considered as passive loads in the magnetosphere-ionosphere coupling models.

One of the most recent arc models is presented by Haerendel (2007, see also earlier references therein) and it is based on the condition that the plasma pressure in the near-Earth plasma sheet has a maximum in the midnight sector. Consequently the magnetospheric plasma flows are directed away from the midnight sector. Magnetic shear stresses build up along the magnetic field lines as the pressure gradient drives their longitudinal convection which is opposed by frictional forces in the lower ionosphere. As the total force balance needs to be maintained in a quasi-stationary situation the regions of strong shear are assumed to cause steep ledges in the radial pressure distribution. The ledges appear as extended structures along the longitudinal convection and at their locations the pressure increases abruptly when going towards lower L-values. At the pressure ledge a localized sheet of FAC (antiparallel to the ambient B-field) builds up and the intensifying FAC sets up favourable conditions for field-aligned potential drops to appear. When such a potential drop appears it decouples the magnetospheric and ionospheric convection and consequently the magnetic shear stress along the field line gets released. Part of the released energy is converted to the kinetic energy of auroral particles in the acceleration region. The model of Haerendel postulates that the front of stress release propagates towards regions of higher magnetic shear (i.e. typically towards lower L-values), but this motion is not frozen with the ambient plasma convection. 
The arc model of Haerendel (2007) is upgraded in the continuation work by Haerendel (2008) where an auroral arc acts as a current generator (or transformer) in the M-I current circuit. This can happen when the ambient electric field has a component tangential to the arc. The tangential electric field causes Hall currents flowing across the arc and concomitant polarization electric field. Haerendel investigates the conditions for the meridional current diversion to FACs (transformer currents) at the arc edges and formulates a quantitative relationship between the background tangential electric field and the relative strength of the transformer current. The model assumes the upward transformer current to be balanced by a localized downward current which in the evening sector is located in the equatorward edge of the arc. The energy related with the transformer currents gets partly deposited in the auroral acceleration region but it may affect also dynamics of the pressure ledge in the magnetosphere and consequently reduce the equatorward propagation velocity of the arc.

Getting observational evidence to evaluate theoretical arc models is a challenging task since simultaneous data should be available from at least three separate key regions: magnetospheric equator, the auroral acceleration region and the ionospheric footpoint where the arc resides. In this paper we provide some optical, radar and low-altitude satellite (Demeter) observations to evaluate some aspects of Haerendel's arc model. With the combination of Demeter particle observations and ground-based optical data we are able to address the contribution of the ionospheric plasma in the arc dynamics and discuss the active link between the ionosphere and the region of field-aligned potential drop at the altitude of few thousands $\mathrm{km}$. We also study the hypothesis presented in Haerendel (1999) which suggests that the cause for arc structures with scale sizes less than tens of $\mathrm{km}$ are ions upwelling from the leading edge of the propagating arc and modifying the potential distribution in the region of field-aligned potential drop and thus producing an intensification of the downward electron flow in a confined region.

The structure of this paper is the following. We start with descriptions of instruments that are used in our analysis (Sect. 2) and of geomagnetic conditions during our event (Sect. 3.1). We continue by analysing the large- and smallscale current system (Sect. 3.2). In Sects. 3.3 and 4 we deduce and discuss the proper motion of the arc with respect to the plasma flow (Sects. 3.3.1, 3.3.2 and 4.1), investigate the dynamics of the structures that make up the arc and analyse perturbations of plasma density and temperature across the current layers associated with the arc (Sects. 3.3.3, 4.2 and 4.3). The main findings are summarised in Sect. 5.

\section{Instrumentation}

The Finland SuperDARN-CUTLASS HF coherent scatter radar (Lester et al., 2004) which is located at geographic co- ordinates $\left(62.3^{\circ} \mathrm{N}, 26.6^{\circ} \mathrm{E}\right)$ covers most of the auroral and polar regions over Scandinavia. The radar operates in the frequency range from 8 to $20 \mathrm{MHz}$. The transmitted waves can be scattered at heights between 100 and $400 \mathrm{~km}$ from fieldaligned density perturbations. The received signal is gated into 75 range gates along 16 beams. Each range gate covers approximately $45 \mathrm{~km}$ in the horizontal plane at the altitude of the wave scattering and the angular separation between adjacent beam directions is $3^{\circ}$. The parameters deduced from the autocorrelation function of the received signal are the velocity of electron irregularities along the radar beam, the scattered power and the spectral width. The temporal resolution of the measurements which is $2 \mathrm{~min}$ in survey mode was reduced to 1 min during our campaign.

From 15 to 22 December 2006 the ALFA (Auroral Light Fine Analysis) mobile all-sky camera was installed and operated from the shelter roof of KEOPS (Kiruna Esrange Optical Platform System) scientific base located at geographic co-ordinates $\left(67.87^{\circ} \mathrm{N}, 21.02^{\circ} \mathrm{E}\right)$ (http://superdarn. cetp.ipsl.fr/ALFA/KEOPS.htm). This camera detects the photo-emissions simultaneously in three wavelength ranges from $\sim 400$ to $700 \mathrm{~nm}$ corresponding to the Bayer filters. The images acquired by ALFA have a spatial resolution of $150 \mathrm{~m}$ in the zenith and of $\sim 5 \mathrm{~km}$ in the horizon when assuming auroral structures at the altitude of $150 \mathrm{~km}$. The temporal resolution of the camera is $30 \mathrm{~s}$.

The Demeter satellite is moving on a nearly polar orbit at the altitude of $700 \mathrm{~km}$. The on-board instruments the measurements from which are used in the present paper are the retarding potential ion analyser (APR) and the fluxgate magnetometer. The APR retarding analyser is designed to measure atomic and molecular ions with densities down to a few particles per $\mathrm{cm}^{-3}$ and relative masses up to $\sim 56$. The entrance of the various charged species into the analyser is controlled by the polarisation grids. One of them is polarised at $-12 \mathrm{~V}$ in order to prevent the electrons with energies lower than $12 \mathrm{eV}$ from reaching the collector. A variable positive potential between 0 and $\sim 20 \mathrm{~V}$ is applied to another grid during each sequence of measurements. The variation of the current collected by the analyser versus the grid potential is used to deduce the ion plasma composition, the concentration of each species, the temperature and the velocity component parallel to the analyser axis. A detailed description of the instrument and models used to deduce the plasma parameters can be found in the papers by Séran (2003) and Berthelier et al. (2006).

For monitoring background conditions in the ionosphere we use magnetometers and optical data from the MIRACLE and IMAGE networks (Syrjäsuo et al., 1998). In standard operation mode the time resolution of magnetic observations is $10 \mathrm{~s}$, while the sampling rate of the all-sky cameras is $\sim 20 \mathrm{~s}$. 


\section{Observations}

\subsection{Geomagnetic conditions}

The period considered in this paper started at 19:19UT ( 22:19 MLT at KEOPS) on 19 December 2006 and lasted about $7 \mathrm{~min}$. Although the global magnetic activity level was fairly low during almost the whole day (estimated $3 \mathrm{~h}$ averaged $K_{p}$-index equals 2), IMAGE magnetometers in the northern Fennoscandia recorded a distinct substorm during 18:30-22:30 UT. At the beginning of the substorm (18:3019:40 UT) magnetic deviations were strongest at geographical latitudes around $70^{\circ}$, but later on the activity intensified at the higher latitudes above $\sim 74^{\circ}$. Shown in Fig. 1 are the variations of magnetic field (north component) recorded by IMAGE magnetometers during one hour starting from 19:00 UT. Geographical latitude of each magnetic station is written underneath the corresponding observation. Substorm growth phase is observed simultaneously by the KIL, TRO and SOR magnetometers located between of $69^{\circ}$ and $71^{\circ}$ geo lat and by optical all-sky cameras from the KEOPS and MUO sites (Fig. 2). Southward drifting auroral arcs appeared to the sky simultaneously with the negative deviation in the magnetic north component. At around 19:21:30 UT the substorm region is extended southward to $\sim 67^{\circ}$. This expansion is visible in KIR and PEL magnetograms (green lines in Fig. 1) and appears in the all-sky images as an isolated arc detached southward from the main oval (Fig. 2). The arc is less bright and much thinner than the auroral structures observed inside the auroral oval. A particular feature of this arc is that the intensity of the electron precipitation causing it is sufficiently high to create perturbations in the electron density in the F-region, but low enough not to cause absorption of the HF waves. This opportunity allows us to put together simultaneous optical, wave and plasma observations inside and in the vicinity of the arc in order to study its features and the possible mechanisms of arc generation.

\subsection{Field-aligned currents}

In order to situate the observed auroral structures in the large-scale current circuit we apply the two-dimensional version of SECS (Spherical Elementary Current Systems) method (Amm, 2001). The SECS method calculates the spatial distribution of the equivalent horizontal currents (cf. Fukushima, 1976) which cause the same magnetic perturbations on the ground as the three-dimensional real currents. In this study we estimate the distribution of FACs with the curl of the equivalent current density. The direction and density of the equivalent currents (black arrows) calculated on a uniform grid of geographical coordinates are superposed in Fig. $2 \mathrm{~b}$ with the $557 \mathrm{~nm}$ emissions observed by MUO imager. Note that our FAC and equivalent current estimations assume a uniform distribution of conductivities with the ratio of Hall to Pedersen conductance equal to 2. All parame-

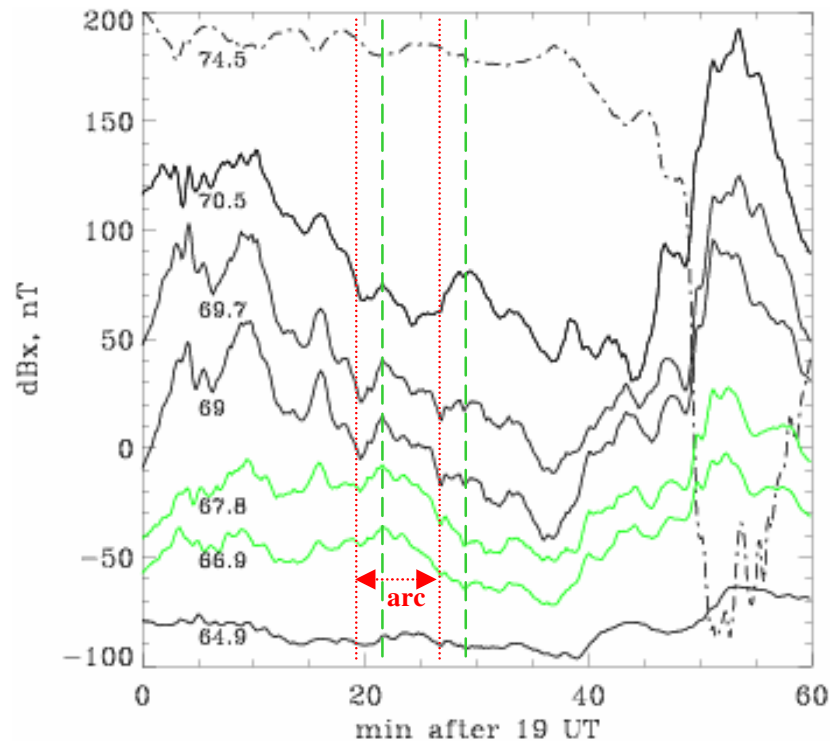

Fig. 1. Variations of magnetic field (north component) recorded by BRJ, SOR, TRO, KIL, KIR, PEL, RVK magnetometers of IMAGE network during one hour starting from 19 UT on 19 December 2006. Geographical latitude of each magnetic station is written underneath the corresponding observation.

ters are mapped to the altitude of $110 \mathrm{~km}$ where most intensive ionospheric currents typically flow. The curl of equivalent currents is then calculated along the line which corresponds to the projection of the Demeter trajectory along the magnetic field lines at the altitude of $110 \mathrm{~km}$. The resulting current density versus geographical latitude is shown in Fig. 3 by the blue dashed line. Positive/negative sign stands for the upward/downward current, respectively. The red line in the same figure corresponds to the currents deduced from the magnetic measurements onboard Demeter using the infinite current sheet approximation (Séran and Cerisier, 2005). The large-scale variations of both current distributions are similar with an upward current around $70^{\circ}$ enclosed by two downward currents. The simplification of assuming homogeneous conductances may be one explanation for the roughly one degree shift in the latitude of peak upward current as deduced from the SECS model with respect to the result from on-board Demeter measurements. The discrete arc at $67.7^{\circ}$ is situated in the region of large-scale downward current, but associated with a meso-scale current layer which carries the upward current. This current is detected by the onboard magnetometer and it can be also deduced from the signal recorded by the APR ion analyser onboard Demeter (black line in the same figure). As explained in our previous paper (Séran et al., 2007), the APR analyser which was designed to measure positively charged species is not protected against the collection of suprathermal electrons with energies higher than $12 \mathrm{eV}$. In the auroral regions, where this population can be significant, the high energy electrons that 

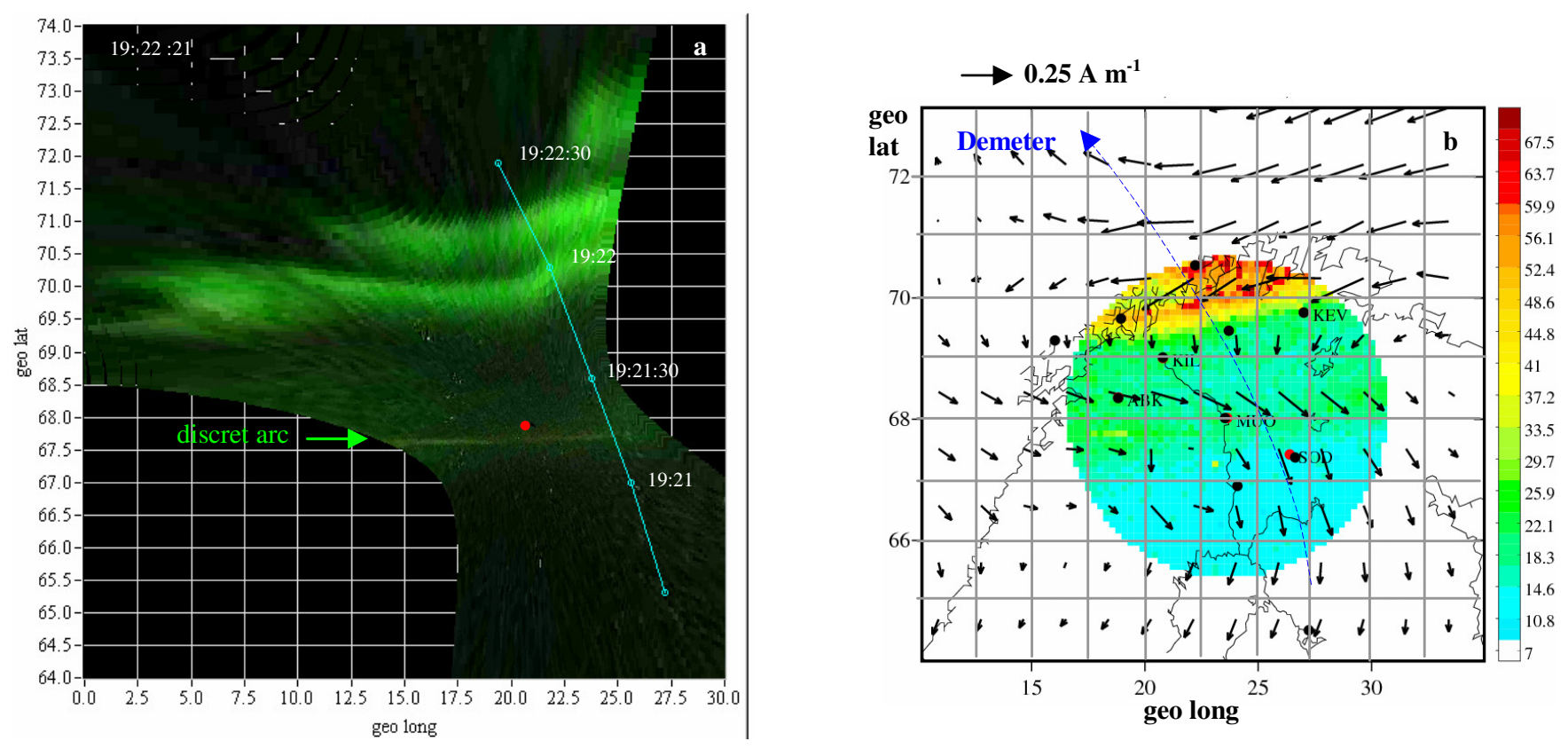

Fig. 2. (a) Wide-band photo-emissions observed by ALFA all-sky camera and (b) $557 \mathrm{~nm}$ emissions recorded by Muo imager superimposed with equivalent currents (black arrows) deduced from the ground-based magnetic measurements. Filled points display geographical location of ALFA all-sky (a) and Image magnetometers (b). Demeter trajectory projected along the magnetic field lines is shown by blue line. All parameters are presented in geographical coordinates and mapped at the altitude of $110 \mathrm{~km}$ and correspond to 19:22 UT on 19 December 2006.

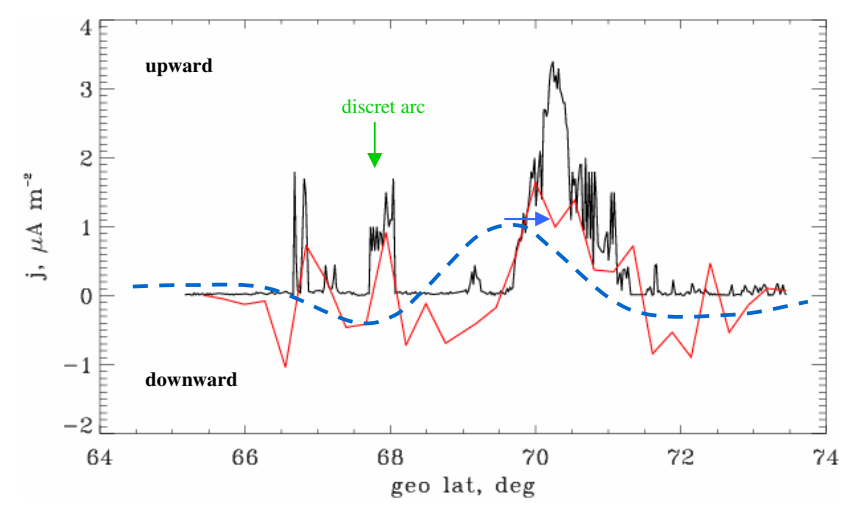

Fig. 3. Field-aligned currents along the Demeter orbit as deduced from the ground magnetic measurements (blue dashed line), from the onboard magnetic (red line) and particle (black line) observations. Positive/negative sign stands for upward/downward current. Note that sensitivity of onboard fluxgate magnetometer is $\sim 3 \mathrm{nT}$.

reach the analyser create a negative current which compensates the current carried by the minor (and sometimes even by major) ion species. We note that each enhancement of the electron current deduced from the retarding analyser is associated with an upward current seen by the onboard magnetometer. Observed differences in the current amplitudes can be explained by at least two reasons. First, the magnetic perturbations that are used to deduce the total current are not necessarily caused only by the local current structure, but they reflect an ensemble of the near-by currents. In contrast, the negative current collected by the ion analyser is only due to the local electron flow that reaches the collector. Second, the angle between the analyser axis and the magnetic field varies from $85^{\circ}$ to $75^{\circ}$ during the considered period. Having an acceptance angle of $106^{\circ}$ the analyser will collect the electrons with a velocity vector which consists an angle larger than $22^{\circ}$ with respect to the magnetic field direction. Therefore the collected negative current contains two components, i.e. the first is due to the field-aligned electron flow and the second is due to the field-perpendicular motion. This second component does not contribute to the total current, since in the case of an isotropic particle distribution in the plane perpendicular to the magnetic field, it is compensated by the opposite electron motion.

Figure 4 shows the upward current deduced from APR together with the optical emission intensities in r-g-b channels recorded by ALFA imager along the satellite pass. This Figure demonstrates that each layer of upward current corresponds to an optical arc, with the exceptions of two thin current structures just below $\sim 67^{\circ}$ which were probably related with a weak increase of photoemissions just above $67^{\circ}$. Such a disagreement in latitude between the optical and current data can be explained by a displacement of emission layer to some higher altitudes than used as default in our mapping. 


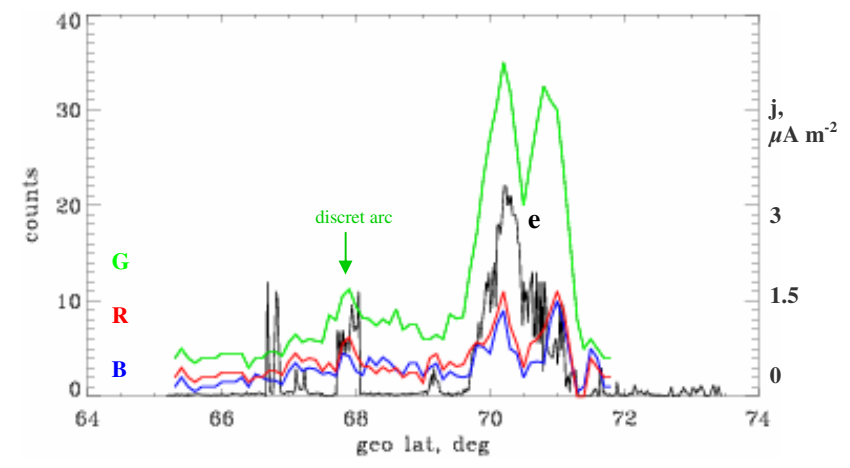

Fig. 4. Photo-emissions observed by ALFA imager in r-g-b channels superposed with the upward currents observed by APR along the Demeter pass.

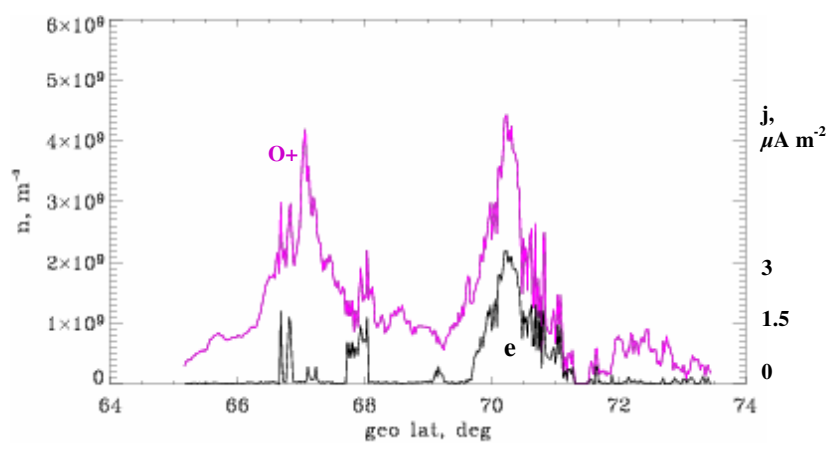

Fig. 5. $\mathrm{O}^{+}$density superimposed with the upward current observed by APR along the satellite pass.

\subsection{Arc and plasma dynamics}

\subsubsection{Arc motion}

During the considered time period the Hankalsami CUTLASS HF radar received echoes along almost all azimuth directions which are localised in one or maximum two adjacent range gates. Two observation sequences starting at 19:22 and 19:27 UT (Fig. 6) illustrate an estimated position of density perturbations from which the HF power was scattered and the temporal evolution of the backscatter region. The region of power scattering represents a layer with large longitudinal extension and narrow latitudinal width which moves towards the South. In the five minutes that separate the two observations the layer was displaced toward the radar by about $45 \mathrm{~km}$ (size of one gate). In order to estimate the temporal variation of the sheet speed we first determine the layer position along each beam direction, assuming that it corresponds to a centre of mass, $r_{k}^{*}$, of the density perturbations, $\Delta n_{k j}$, i.e. $r_{k}^{*}=\sum_{j} r_{k j} \Delta n_{k j} / \sum_{j} \Delta n_{k j}$, here $r_{k j}$ is the central position of the j-gate along the k-beam. Since the amount of energy scattered from the field-aligned density irregularities is proportional to the squared density perturba-

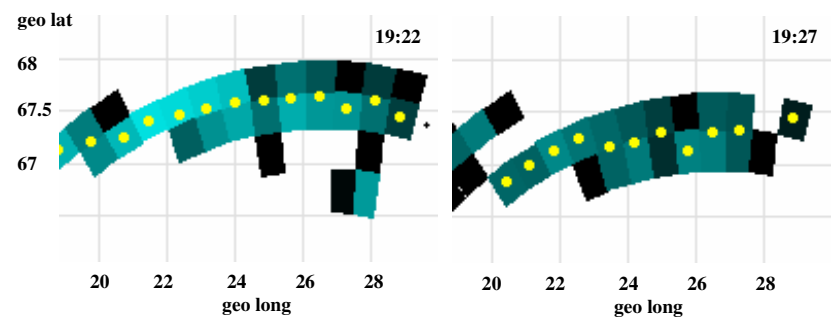

Fig. 6. Geographical location of the regions of HF power scattering. Yellow points correspond to the centre of mass of the density perturbations along each radar beam. Colour represents the HF power.

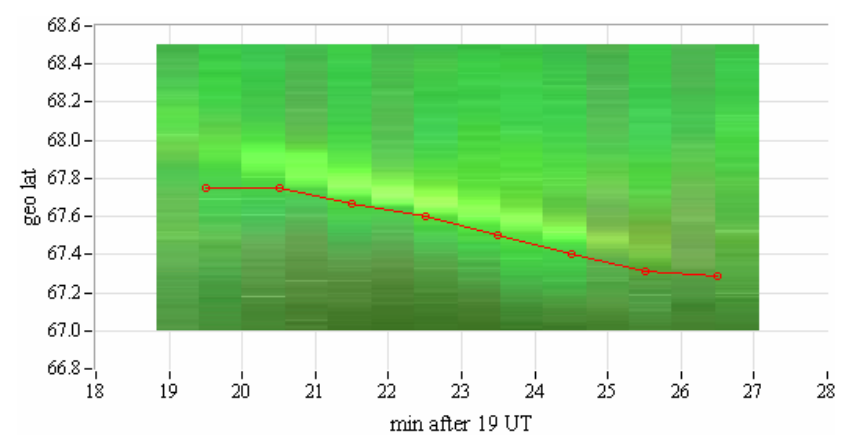

Fig. 7. Latitudinal distribution of light emission versus time, deduced from the ALFA images. Each value represents an average emission in the longitudinal range between 14 and $27^{\circ}$. Bright spot corresponds to the discrete arc which is moving southward. Red circles show estimated central position of layer which scatters the HF waves.

tion (cf. Minkoff, 1974), the above expression can be rewritten in terms of scattered power $P_{k j}$, expressed in $\mathrm{dB}$. Thus, $r_{k}^{*}=\sum_{j} r_{k j} 10^{0.05 P k j} / \sum_{j} 10^{0.05 P k j}$. Yellow points in the Fig. 6 show the layer position along each direction, calculated according to the above method. The temporal variation of the layer position in the longitudinal sector $\left(24^{\circ}, 26^{\circ}\right)$ is then shown in Fig. 7 by red circles.

Independently, we compute and display in the same Fig. 7 the latitudinal distribution of emissions recorded by the allsky camera. Each value represents an average emission calculated in the longitudinal range between 14 and $27^{\circ}$ in r-g-b channels. Intense spot in each column corresponds to the discrete arc, which progressively moved southward. The displacement is $\sim 0.05^{\circ}$ for each $35.5 \mathrm{~s}$. This corresponds to a velocity of $\sim 150 \mathrm{~m} \mathrm{~s}^{-1}$.

The remarkable consistency between the position and displacement of the arc and that of the layer of the HF backscatter demonstrates that the assumption that HF waves were scattered from the region of electron precipitation is likely to be correct.

Another important observation is the correlation between the arc brightness and its speed. At the beginning and at the end of shown sequence when the arc intensity is weak its speed (in the earth frame) vanishes. 

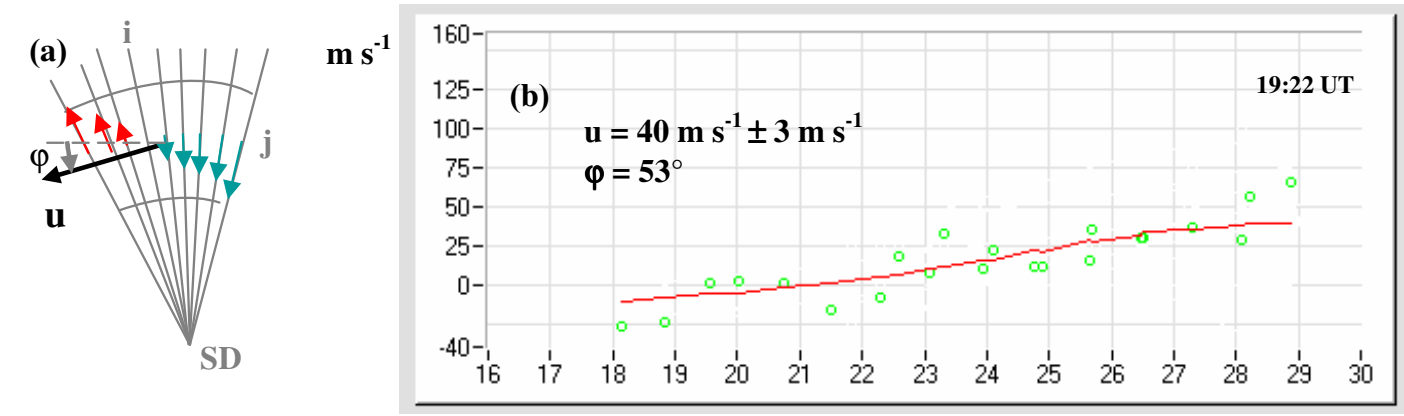

geo long

Fig. 8. (a) Sketch that illustrates projection of plasma velocity along the radar beams. (b) Beam-aligned plasma velocities calculated from Doppler shift of HF signal (green circles) and the fit (red line) which allows to deduce the velocity vector in the plane perpendicular to magnetic field.

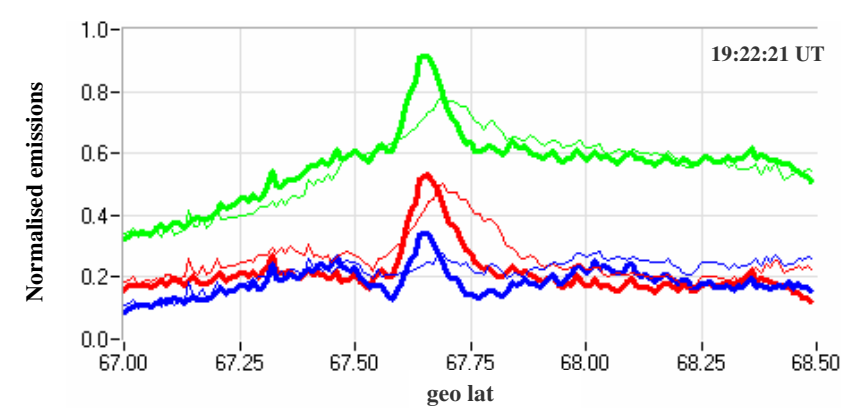

Fig. 9. Latitudinal distribution of normalised r-g-b emissions across the arc averaged in the two longitudinal sectors, i.e. between $14^{\circ}$ and $20.5^{\circ}$ (thick lines) and $20.5^{\circ}$ and $27^{\circ}$ (thin lines).

\subsubsection{Plasma motion}

Our next step is to check if the plasma flow follows the arc motion? In order to deduce the plasma velocity from the HF measurements conducted from one single site we have to assume that the average velocity vector is uniform along the scattering layer of large longitudinal extension. Of course this hypothesis is not valid in the cases of flow rotation or shear. The validity of this method can be justified for each application by analysing the deviation of the observed values with respect to the estimated ones. In this approach, two parameters that characterise the average velocity, i.e. its amplitude, $u$, and azimuth angle, $\varphi$, are estimated using the least squares method, which consists in minimising of a function written in the form $f=\sum_{i, j}\left[v_{i j}-u\left(\cos \varphi \cos \alpha_{i}+\sin \varphi \sin \alpha_{i}\right)\right]^{2}$, where $v_{i j}$ is the velocity measured along each $i$-beam in $j$-range, and $\left(\cos \alpha_{i}\right.$, $\sin \alpha_{i}$ ) is the unit vector along $i$-beam. An example of beamaligned velocities observations (green circles) and their fit (red line) is presented in Fig. 8. In the considered example, the average velocity is estimated about $40 \mathrm{~m} \mathrm{~s}^{-1}$ and the flow direction measured anticlockwise from geographic West is $\sim 53^{\circ}$. The error in the estimation of these parameters is about $3 \mathrm{~m} \mathrm{~s}^{-1}$ in amplitude or $3^{\circ}$ in azimuth. During the whole period between 19:20 and 19:30 UT the plasma velocity varied between 40 and $60 \mathrm{~m} \mathrm{~s}^{-1}$ and the azimuth angle corresponded to directions in South - South-West. Thus, the deduced plasma velocity is at least 3 times lower than the arc speed observed by the all-sky camera and by HF radar and implies a westward component in the ionospheric electric field.

\subsubsection{Arc structure}

The part of the arc observed by the ALFA imager (Fig. 2a) extended in longitude from $14^{\circ}$ to $27^{\circ}$ (this corresponds to a length of about $560 \mathrm{~km}$ ) and the arc was almost aligned in the east-west direction. Figure 9 shows the latitudinal distribution of r-g-b emissions averaged in two longitudinal sectors, i.e. between $14^{\circ}$ and $20.5^{\circ}$ (thick lines) and $20.5^{\circ}$ and $27^{\circ}$ (thin lines). If we define the arc width as latitudinal size of the layer inside of which the emission level exceeds the average between maximum and background emissions, then its angular size is found to be $0.1-0.2^{\circ}$. This corresponds to a width of $\sim 10-20 \mathrm{~km}$. Note that the maximum intensity is shifted southward with respect to the geometrical centre of the emission layer, i.e. toward the arc leading edge. The ratio of the distance between the front edge and maximum to the distance between the maximum and the trailing edge is $\sim 2: 3$.

The distribution of emission intensity along the arc was highly variable with dominant structures having a longitudinal size of $0.5^{\circ}$, which corresponds to the length of $\sim 20 \mathrm{~km}$, and the brightness variations of $40-80 \%$ with respect to the average brightness level inside the arc (dashed line in the Fig. 10). The example of two consecutive intensity profiles along the arc separated by $36 \mathrm{~s}$ presented in Fig. 10 demonstrates a displacement of bright structures along the arc. Finding any systematics in the displacements is difficult at the first glance, but at least one can say that there is a 


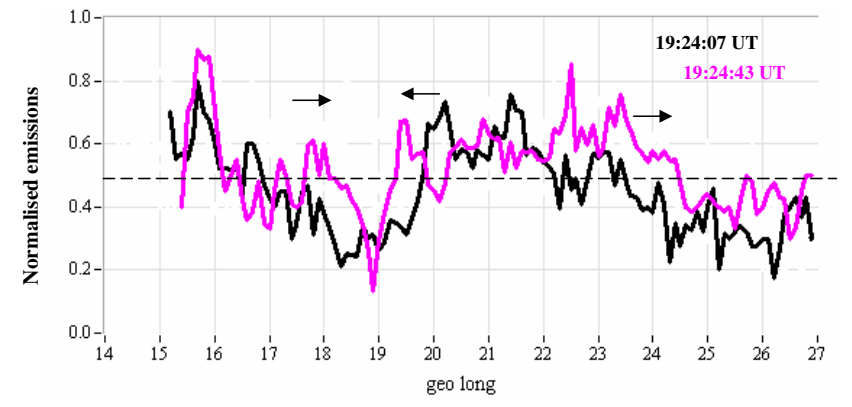

Fig. 10. Variation of red emissions along the arc shown for two consecutive images.

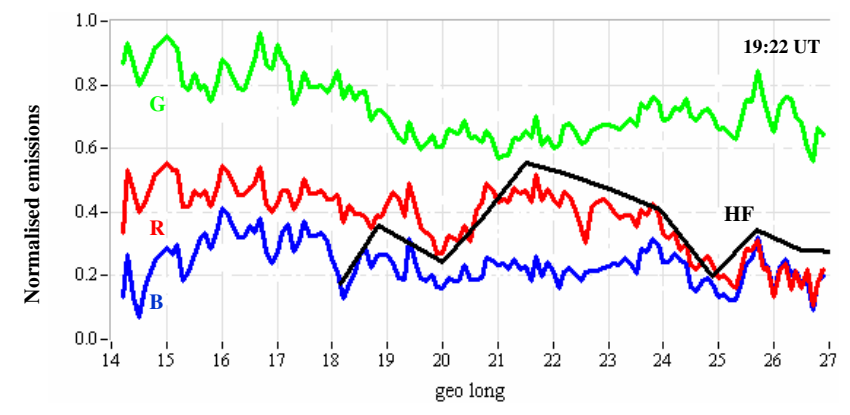

Fig. 11. Variation of r-g-b emissions and HF power along the arc.

tendency for the bright structures to shift into the regions of previously low brightness.

Finally, we compare the optical emissions and HF radar echo associated with the arc. Figure 11 demonstrates that large-scale intensifications of the emissions in the red channel have best correlation with the amplitude variations of HF power received by the radar. This result is not completely surprising if the dominant emission in the red channel is OI $630 \mathrm{~nm}$ emission, since the anticipated altitude of the most efficient wave scattering and that of the OI $630 \mathrm{~nm}$ intensification is approximately the same, i.e. $\sim 200 \mathrm{~km}$. The observed correlation represents some evidence for a close relation between large-scale perturbations generated by precipitating electrons and small-scale plasma irregularities with characteristic size of $\sim 10 \mathrm{~m}$ which scatter the HF waves. In the upper ionosphere, i.e. at the altitudes above $\sim 200$ $300 \mathrm{~km}$, such irregularities of the electron density are generated by the particle transport across the density and temperature gradients (Gurevich, 1978). In our case significant gradients can be anticipated to exist both at the leading edge of the arc and inside it.

\section{Discussion}

The arc described above has several interesting features from the viewpoint of the theoretical arc models by Haerendel (2007, 2008, and references therein). The arc was observed

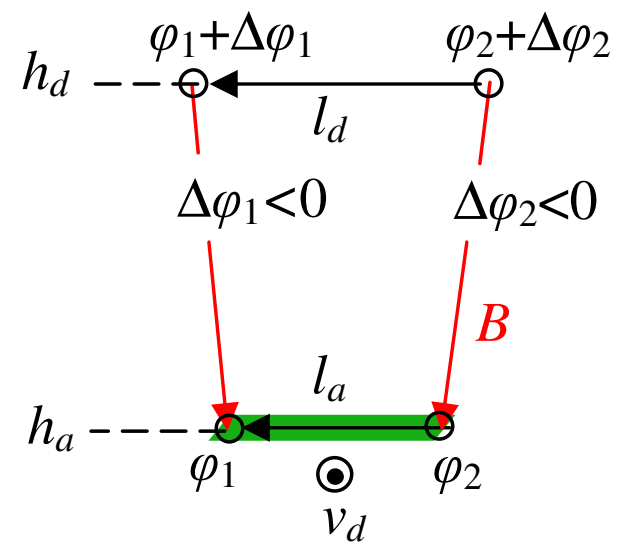

Fig. 12. Schema which illustrates the distribution of electric potentials along the magnetic lines associated with the arc.

in the midnight sector where the total pressure should have its maximum and significant plasma flow perpendicular to the arc was recorded by the CUTLASS radar. Thus at least two basic conditions for a generator-type of arc as suggested by Haerendel were fulfilled in our case. On the other hand, it is important to remember that the arc was observed during a substorm expansion phase and at latitudes equatorward of the main activity region. Consequently, we can expect the arc to have also features non-consistent with theoretical models which usually assume relatively slow and linear evolution like in the case of a substorm growth phase auroral arc.

Below we discuss our observations in the context of the Haerendel arc models. The coupling between ionospheric and auroral acceleration region processes is addressed with the help of DEMETER particle observations.

\subsection{Difference in the arc and plasma flow speeds}

A relative difference between the velocity of auroral structures and ionospheric plasma has been reported in some previous studies which have used incoherent EISCAT radar and optical data (see, for instance, Haerendel et al., 1993; Williams et al., 1998; del Pozo et al., 2002). By analysing the relation between the substorm phases and the arcs dynamics, del Pozo et al. (2002) found that the mismatch in the arc and plasma velocities is usually observed during the expansion phase of the substorm. The arc of our study which moved southward at least three times faster than the surrounding ionospheric plasma was also observed during the substorm expansion phase. The arc appears $\sim 3$ min before the southward expansion of the substorm region recorded by the IMAGE magnetometers (Figs. 1 and 7) and is visible during $\sim 7 \mathrm{~min}$, which is an approximate duration of the expansion phase.

Haerendel et al. (1993) proposed two mechanisms in order to explain the proper motion of arc. The first is related to the motion of the generator with respect to the plasma frame. In 
this case the frozen-in conditions are broken already at the generator level, i.e. in the plasma sheet region. The second mechanism considers the energy balance between the generator in the plasma sheet and the auroral acceleration region (region of field-aligned potential drop, the fracture region) at the altitude of several thousands kilometres. If the amount of energy released into the plasma acceleration in the fracture region is larger than the energy which is pumped from the generator into the electric circuit, then the circuit is forced to shrink. In the opposite situation, the circuit will expand. Upward field-aligned current associated with an evening arc is closed by its return current flowing southward from the arc. Following the second mechanism, arc motion presented in the Fig. 7 corresponds to the shrink circuit.

In both scenarios the difference between plasma velocity and arc motion is a consequence of the discontinuity of the electric field component perpendicular to the magnetic field which likely happens in the region of field-aligned potential drop. Such situation is illustrated in Fig. 12. At each altitude, i.e. below $\left(h_{a}\right)$ and above $\left(h_{d}\right)$ the potential drop $(\Delta \phi)$, the component of the electric field along the arc is deduced to be $E_{a}=-\left(\varphi_{1}-\varphi_{2}\right) / l_{a}$ and $E_{d}=E_{a} l_{a} / l_{d}-\left(\Delta \varphi_{1}-\Delta \varphi_{2}\right) / l_{d}$, respectively. Here $\phi_{1}, \phi_{2}$ and $\phi_{1}+\Delta \phi_{1}, \phi_{2}+\Delta \phi_{2}$ are the electric potentials associated with two magnetic lines separated at distances $l_{a}$ and $l_{d}$ at the heights of $h_{a}$ and $h_{d}$, respectively. Assuming that plasma moves under the action of the $\boldsymbol{E} \times \boldsymbol{B}$ force, the ratio of velocities at each height can be written as $v_{d} / v_{a}=\left(E_{d} / E_{a}\right)\left(B_{a} / B_{d}\right)$. Taking into account that the arc is longitudinally aligned, i.e. $l_{a} / l_{d} \approx\left(R_{E}+h_{a}\right) /\left(R_{E}+h_{d}\right)$, and that the magnetic field strength $B$ decreases with height as $\sim\left(R_{E}+h\right)^{-3}$, the altitude $h_{d}$ above the potential drop is estimated as following $h_{d} \approx R_{E}\left\{\left(1+h_{a} / R_{E}\right) \sqrt{v_{d} / v_{a}} / \sqrt{1-\left(\Delta \varphi_{1}-\Delta \varphi_{2}\right) /\left(l_{a} E_{a}\right)}-1\right\}$, here $R_{E} \approx 6400 \mathrm{~km}$ is Earth's radius. In the case when the potential drop is nearly constant along the arc, i.e. $\Delta \phi_{1} \approx \Delta \phi_{2}$, this altitude depends only on the plasma and arc velocities at the altitude below the discontinuity layer. Substituting $h_{a} \approx 200 \mathrm{~km}$ and $v_{d} / v_{a} \approx 3$ we then get $h_{d} \approx 5000 \mathrm{~km}$.

At such heights the electric antennas onboard the Polar satellite measured the parallel electric fields that exceed $100 \mathrm{mV} \mathrm{m}^{-1}$ (Mozer and Kleitzing, 1998). Such heights are also referenced in the literature as an upper boundary for the Alfvén resonator (see for example, Lysak, 1993). Once launched the Alfvén wave can be trapped and amplified between the ionosphere and this upper boundary which corresponds to the heights where the density of cold ionospheric plasma that exponentially drops with altitude attains the density of the warm magnetospheric plasma. Low beta plasma that fills the regions surrounding the upper boundary is characterised with the electron inertial length of few $\mathrm{km}$. Thus, a component of electric field parallel to the magnetic field might be significant in this region (see for example Kolesnikova et al., 2002).

\subsection{Erosion in the leading edge of the arc}

The emission intensity profile across the observed arc shows a steeper decrease in the southward edge of the arc than in its poleward side (Fig. 9). Erosion of the leading edge of a propagating arc and the formation of ion tongues were considered by Haerendel et al. (1993) and Haerendel (1999) as a promising mechanism to explain a localised enhancement of the field-aligned potential drop in the energy conversion region and consequent electron acceleration toward the ionosphere. This mechanism is working if the time that the ions take to move from the ionosphere to the fracture region is less than the time during which the arc is displaced at the distance equal to its width. In the considered case the characteristic time of the arc displacement in the plasma frame is $\sim 2-3$ min. The average speed of upgoing $\mathrm{O}^{+}$observed by Demeter at $700 \mathrm{~km}$ in the 21:00 and 24:00 MLT sectors in the regions of field-aligned currents varies between 200 and $1000 \mathrm{~m} \mathrm{~s}^{-1}$ (Séran et al., 2007). Obviously, this speed is too slow to reach the altitude of $\sim 5000 \mathrm{~km}$ in few minutes.

How would the above described situation change if the ions moved in the field of an Alfvén wave, which in the plasma with low $\beta$ carries the electric field parallel to the magnetic field? Following the results of our previous paper (Kolesnikova et al., 2002), we assume an exponential variation of the field-aligned electric field with the height, i.e. $E(z)=E_{0} e^{\left(z-z_{0}\right) /(2 h)}$, between the topside ionosphere and upper boundary of the Alfvén resonator, i.e. $z_{0} \leq z \leq z_{1}$. Here $h$ is the characteristic height; $E_{k}$ and $u_{k}$ are, respectively, the fieldaligned electric field and speed at the height $z_{k}$. Then the field-aligned velocity varies as $u^{2}=u_{0}^{2}+a_{0}\left(e^{\left(z-z_{0}\right) /(2 h)}-1\right)$ with $a_{0}=4 \mathrm{em}_{i}^{-1} E_{0} h$ and the time of flight is estimated to be $t=\int_{z_{0}}^{z_{1}} \frac{d z}{u}=\left.\frac{2 h}{\sqrt{u_{0}^{2}-a_{0}}} \ln \left|\frac{u+\sqrt{u_{0}^{2}-a_{0}}}{u-\sqrt{u_{0}^{2}-a_{0}}}\right|\right|_{u_{1}} ^{u_{0}}$ for $u_{0}^{2}>a_{0}$ or $\left.\frac{4 h}{\sqrt{-u_{0}^{2}+a_{0}}} \operatorname{arctg}\left[\frac{u}{\sqrt{-u_{0}^{2}+a_{0}}}\right]\right|_{u_{0}} ^{u_{1}}$ for $u_{0}^{2}<a_{0}$. The variation of the flight time versus amplitude of field-aligned electric field at the height $z_{1}$ is shown in the Fig. 13 for two ion species, i.e. $\mathrm{O}^{+}$(in red) and $\mathrm{H}^{+}$(in blue), in the conditions with $h=450 \mathrm{~km}, z_{0}=700 \mathrm{~km}, z_{1}=5000 \mathrm{~km}$ and $u_{0}=1000 \mathrm{~m} \mathrm{~s}^{-1}$. Thus, in the considered case, the ions outgoing from the ionosphere will potentially contribute to the energy re-distribution inside the fracture region if the fieldaligned electric field on the bottom side of this region is around $0.01 \mathrm{mV} \mathrm{m}^{-1}$ for $\mathrm{H}^{+}$and a few $0.1 \mathrm{mV} \mathrm{m}^{-1}$ for $\mathrm{O}^{+}$.

Variation of ion temperature across the arc deduced from the APR observations on board Demeter demonstrates a significant ion cooling at the leading edge of the propagating arc. The $\mathrm{O}^{+}$temperature shown by crosses in Fig. 14 decreases by a factor of two in the $5 \mathrm{~km}$ layer adjacent to the arc. Note that the absence of measurements between $67.75^{\circ}$ and $68.05^{\circ}$ is due to an abundance of suprathermal 


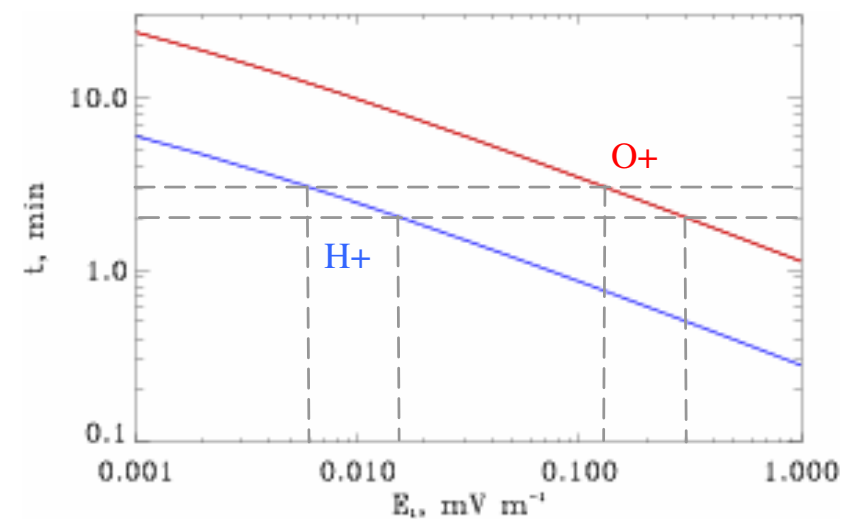

Fig. 13. Time flight of $\mathrm{O}^{+}$(red) and $\mathrm{H}^{+}$(blue) between 700 and $5000 \mathrm{~km}$ in the field of Alfvén wave versus amplitude of the fieldaligned electric field at $5000 \mathrm{~km}$. Electric field is assumed to increase exponentially with altitude; characteristic height $h$ is fixed at $450 \mathrm{~km}$ and the upward velocity at $700 \mathrm{~km}$ altitude is taken to be $1000 \mathrm{~m} \mathrm{~s}^{-1}$.

electrons in the region of electron precipitation (cf. discussion in Sect. 3.2).

The observed cold ion population at the leading edge of the arc is likely outgoing flow of $\mathrm{O}^{+}$which is newly produced at the lower latitudes and pushed away from the layer of the electron precipitation by increased plasma pressure and then upward by relative motion arc-plasma. The positive density gradient along the direction of arc propagation (cf. Fig. 5) creates additional force in favour of $\mathrm{O}^{+}$upward motion. This mechanism is quite similar to that proposed by Séran et al. (2007) to explain the outgoing of cold $\mathrm{O}^{+}$ions from multi-layers of field-aligned currents.

\subsection{Intensity variations along the arc}

The brightness was not homogeneous along the arc. The emission intensity varied up to $40-80 \%$ with respect to the average level inside the arc. We suggest that a plausible mechanism to control the observed restructuring inside the arc is the outflowing of ionospheric ions. If we assume that such bright spots are associated with an increase of potential $\operatorname{drop} \delta \varphi$ in the fracture region and that emission intensity is proportional to the flow of precipitating electrons, then resulted emissions varies as $\sim \exp (e \delta \varphi / T)$, here $T$ is the fieldaligned temperature of precipitating electrons and $e$ is an elementary charge. According to the Hardy model (Hardy et al., 1985), the expected temperature in the considered local time sector and in the conditions of magnetic activity, which were observed at 19:00 UT, i.e. $a_{p}=9$, is about $500 \mathrm{eV}$. This gives the estimations of potential variations of $\sim 200-300 \mathrm{~V}$.

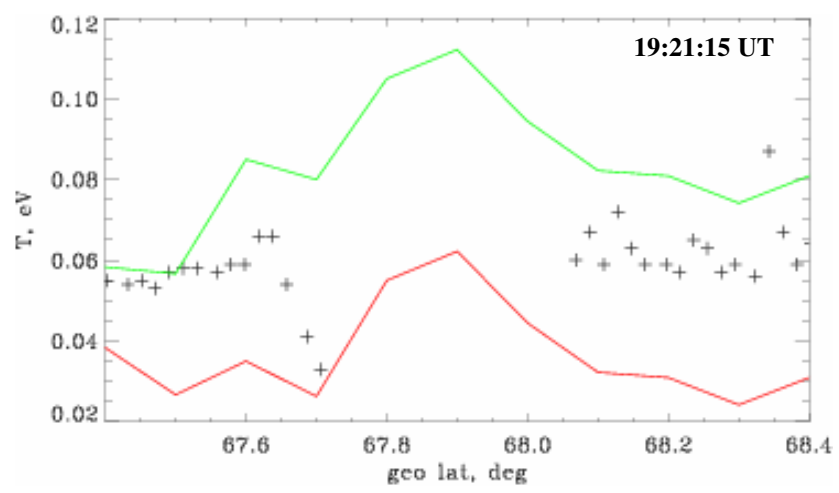

Fig. 14. Variation of $\mathrm{O}^{+}$temperature across the arc measured by ion analyser onboard Demeter (crosses) superposed with the r-g emissions observed by ALFA along the satellite pass.

\section{Conclusions}

We have presented observations of a southward propagating discrete arc detached by $\sim 200 \mathrm{~km}$ southward from the main substorm region. Besides optical observations the arc was visible also as a band of enhanced backscatter in the CUTLASS HF radar data. The arc was observed during substorm expansion phase and had a speed three times higher than the velocity of the background plasma flow. The arc speed was correlated with the arc brightness, i.e. the arc speed (as deduced from the HF backscatter signal) dropped down when the emissions faded away. The arc had several features consistent with the Haerendel arc model (1993, 2007, 2008) where the arc is a consequence of release of magnetic shear stresses which build up in the field lines as their magnetospheric end performs longitudinal convection in the plasma sheet (midnight sector) and their ionospheric end opposes this motion due to frictional forces. The stress release takes place in the fracture region (auroral acceleration region) where potential drop builds up and decouples the magnetospheric and ionospheric arc regions. In the presence of ionospheric electric field tangential to the arc, like in our case, the arc can also serve as a current transformer. Our observations suggest that the outgoing of ionospheric ions from the regions of increased electron precipitations may control the potential distribution inside the fracture region and thus the arc brightness and even its motion. Besides the difference in arc and plasma motion also cold $\mathrm{O}^{+}$observed at the leading edge of the arc could have shifted the maximum emission intensity toward the direction of arc propagation as suggested in the Haerendel arc model. Motion of bright structures along the arc is a further feature in our observations which seems support this picture. Our data set contains ionospheric observations (at altitudes of $\sim 100-200 \mathrm{~km}$ and $\sim 700 \mathrm{~km}$ ). A more comprehensive testing of the model (e.g. according to the guidelines given in Haerendel, 2008) is left for future studies with the hope of finding an event where observations are 
available also from the magnetospheric generator and auroral acceleration regions.

Acknowledgements. We thank our colleges from ESRANGE for their help in organisation of the KEOPS campaign. Special thanks to ALFA and Demeter teams for the high quality work and Liisa Juusola (FMI) for the equivalent current calculations. Mark Lester was supported by STFC award PP/E000983. The ALFA development was funded by PNST/INSU and IPSL. The IMAGE magnetometer data are collected as a joint European collaboration with FMI serving as the PI-institute. The authors thank both referees for constructive criticism.

Topical Editor M. Pinnock thanks M. Echim and another anonymous referee for their help in evaluating this paper.

\section{References}

Amm, O.: The elementary current method for calculating ionospheric current systems from multi-satellite and ground magnetometer data, J. Geophys. Res., 106, 24843-24855, 2001.

Berthelier, J.-J., Godefroy, M., Leblanc, F., Séran, E., et al.: IAP, the thermal plasma analyzer on Demeter, Planet. Space Sci., 54, 487-501, 2006.

Boström, R.: A model of the auroral electrojets, J. Geophys. Res., 69, 4983-4999, 1964.

Fukushima, N.: Generalized theorem for no ground magnetic effect of vertical currents connected with Pedersen currents in the uniform-conductivity ionosphere, Rep. Ionos. Space Res. Japan, 30, 35-40, 1976.

Gurevich, A. V.: Nonlinear phenomena in the ionosphere, edited by: Roederer, J. G. and Wasson, J. T., Springe, 1978.

Haerendel, G., Buchert, S., La Hoz, C., Raaf, B., and Rieger, E.: On the proper motion of auroral arcs, J. Geophys. Res., 98, 60876099, 1993.

Haerendel, G.: Origin and dynamics of thin auroral arcs, Adv. Space Res., 23, 10, 1637-1645, 1999.

Haerendel, G.: Auroral arcs as sites of magnetic stress release, J. Geophys. Res., 112, A09214, doi:10.1029/2007JA012378, 2007.

Haerendel, G.: Auroral arcs as current transformers, J. Geophys. Res., 113, A07205, doi:10.1029/2007JA012947, 2008.

Hardy, D. A., Gussenhoven, M. S., and Holfman, E.: A statistical model of auroral electron precipitation, J. Geophys. Res., 90, 4229-4248, 1985.

Kolesnikova, E., Robinson, T. R., Davies, J. A., Wright, D. M., and Lester, M.: Excitation of Alfvén waves by modulated HF heating of the ionosphere, with application to FAST observations, Ann. Geophys., 20, 57-67, 2002,

http://www.ann-geophys.net/20/57/2002/.
Lester, M., Chapman, P. J., Cowley, S. W. H., Crooks, S. J., Davies, J. A., Hamadyk, P., McWilliams, K. A., Milan, S. E., Parsons, M. J., Payne, D. B., Thomas, E. C., Thornhill, J. D., Wade, N. M., Yeoman, T. K., and Barnes, R. J.: Stereo CUTLASS - A new capability for the SuperDARN HF radars, Ann. Geophys., 22, 459-473, 2004, http://www.ann-geophys.net/22/459/2004/.

Lysak, R. L.: Generalized model of the ionospheric Alfvén resonator, in: Auroral plasma dynamics, edited by: Lysak, R. L., 121-128, AGU, Washington, 1993.

Minkoff, J.: Radio frequency scattering from a heated ionospheric volume, 3, Cross section calculations, Radio Sci., 997-1004, 1974.

Mozer, F. S. and Kletzing, C. A.: Direct observations of large, quasi-static, parallel electric fields in the auroral acceleration region, Geophys. Res. Lett., 25, 1629-1632, 1998.

del Pozo, C. F., Williams, P. J. S., Gazey, N. J., Smith, P. N., Honary, F., and Kosch, M.: Multi-instrument observations of the dynamics of auroral arcs: a case study, J. Atmos. Solar Terr. Phys., 64, 1601-1616, 2002.

Rothwell, P. L., Silevitch, M. B., Block, L. P., and Fälthamar, C.-G.: Prebreakup arcs: A comparison between theory and experiment, J. Geophys. Res., 96, 13967-13975, 1991.

Séran, E.: Reconstruction of the ion plasma parameters from the current measurements: mathematical tool, Ann. Geophys., 21, 1159-1166, 2003, http://www.ann-geophys.net/21/1159/2003/.

Séran, E. and Cerisier, J.-C.: Current layers: influence of the finite size and non-uniform current distribution, J. Atmos. Solar Terr. Phys., 67, 729-737, 2005.

Séran, E., Frey, H. U., Fillingim, M., Berthelier, J.-J., Pottelette, R., and Parks, G.: Demeter high resolution observations of the ionospheric thermal plasma response to magnetospheric energy input during the magnetic storm of November 2004, Ann. Geophys., 25, 2503-2511, 2007, http://www.ann-geophys.net/25/2503/2007/.

Syrjäsuo, M. T., Pulkkinen, T. I., Janhunen, P., Viljanen, A., Pellinen, R. J., Kauristie, K., Opgenoorth, H. J., Wallman, S., Eglitis, P., Karlsson, P., Amm, O., Nielsen, E., and Thomas, C.: Observations of substorm electrodynamics using the MIRACLE network, in Substorms-4, edited by: Kokubun, S. and Kamide, Y., Terra Scientific Publishing Company, Tokyo, 111-114, 1998.

Williams, P. J. S., del Pozo, C. F., Hiscock, I., and Fallows, R.: Velocity of auroral arcs drifting equatorward from the polar cap, Ann. Geophys., 16, 1322-1331, 1998, http://www.ann-geophys.net/16/1322/1998/. 